\title{
$\beta 3$-integrin Leu33Pro gain of function variant does not modulate inflammatory activity in human derived macrophages in diabetes
}

\author{
Philipp Helmer ${ }^{\circledR}$, Ellen Damm¹, Stephan Schiekofer ${ }^{2,3}$, Kirsten Roomp ${ }^{4}$, Jochen G. Schneider ${ }^{1,4}$ \\ 1. Saarland University, Medical Center, Dpt. of Internal Medicine II, Homburg, Saar, Germany. \\ 2. Zentrum für Altersmedizin, Klinik und Poliklinik für Psychiatrie und Psychotherapie der Universität Regensburg am Bezirksklinikum, Regensburg, \\ Germany. \\ 3. Sigmund Freud Privat-Universität, Wien, Austria. \\ 4. Luxembourg Centre for Systems Biomedicine (LCSB), University of Luxembourg, Belvaux, Luxembourg, and Centre Hospitalier Emile Mayrisch, \\ Esch/Alzette, Luxembourg. \\ $\square$ Corresponding authors: Jochen G. Schneider, Luxembourg Centre for Systems Biomedicine (LCSB), Campus Belval, 6 Ave. du Swing, L-4367 Belvaux, \\ Luxembourg, Ph: 00352 4666446154, Fax: 00352 46664436154, Email: jg.schneider@outlook.com. Philipp Helmer, current address: Department of Anesthesiology \\ and Critical Care, University Hospital Wuerzburg, Oberduerrbacher Strasse 6, 97080 Wuerzburg, Germany
}

(1) The author(s). This is an open access article distributed under the terms of the Creative Commons Attribution License (https://creativecommons.org/licenses/by/4.0/). See http://ivyspring.com/terms for full terms and conditions.

Received: 2020.11.09; Accepted: 2020.12.21; Published: 2021.05.13

\begin{abstract}
Objective: We aimed to investigate the association between the Leu33Pro ( $r 55918$ ) polymorphism in $\beta 3$-integrin with diabetic complications and inflammatory function of macrophages depending on the genotype in subjects with diabetes mellitus.

Material and methods: We determined the Leu33Pro polymorphism in 186 diabetic subjects and collected laboratory data. Monocytes from 24 patients were collected for macrophage differentiation to determine the inflammatory activity by treating with different stimulants.

Results: We could demonstrate that human derived differentiated macrophages expressed $\beta 3$-integrin. Their secretory capacity upon inflammatory stimulation did not reveal any differences depending on the Leu33Pro variant. We found trends for an association of the polymorphism with the presence of diabetic nephropathy $(p=0.071)$, as well as with creatinine $[1.32 \mathrm{mg} / \mathrm{dL}(1) \mathrm{vs} .0 .98 \mathrm{mg} / \mathrm{dL}(0)](p=0.029$ in recessive model) and glomerular filtration rate $[75.6 \mathrm{ml} / \mathrm{min} \pm 22 \mathrm{vs} .62 .3 \mathrm{ml} / \mathrm{min} \pm 25](p=0.076$ in recessive model) as quantitative markers of kidney function.

Conclusion: Despite the expression of $\beta 3$-integrin in human macrophages, the Leu33Pro polymorphism in $\beta 3$-integrin does not modify the inflammatory response upon stimulation but might play a role in the progression of diabetic nephropathy. Further studies are necessary to substantiate such a hypothesis.
\end{abstract}

Key words: Leu33Pro polymorphism, rs5918, diabetes, diabetic nephropathy, inflammation, macrophages

\section{Introduction}

av $\beta 3$-integrin (aka vitronectin receptor) is a heterodimeric cell surface receptor with varying functions depending on the level and location of its cellular expression. It is involved in signaling both between cells, and between cells and the extracellular matrix [1]. av $\beta 3$-integrin ligation induces conformational changes that lead to the facilitation of signaling events within the cells with consequences such as migration, proliferation, and differentiation [2]. Gene-knockout studies in mice suggested a role for av $\beta 3$-integrin in disease conditions such as inflammation, bone diseases and cancer [3-5]. Consequently, $\beta 3$-integrin modulation, as a part of the vitronectin receptor, has been tested to reduce osteoporosis, tumor growth and angiogenesis by blocking $\beta 3$-integrin with antibodies or RGD peptide antagonists, yet the results are inconsistent. In clinical studies a natural modulation of $\beta 3$-integrin by the Leu33Pro polymorphism (rs5918) has been demonstrated to be associated with diabetes mellitus 
and furthermore diabetic vascular diseases $[6,7]$. The mechanism of this observation remains unclear so far. However, the Leu33Pro polymorphism results in a constitutively active $\beta 3$-integrin [8] and $\beta 3$-integrin-deficient macrophages have been shown to be involved in inflammatory atherosclerosis [3].

The aim of our study was to investigate if there is an association between the Leu33Pro polymorphism with diabetic complications and to functionally characterize the inflammatory activity of human differentiated macrophages in carriers of the polymorphism versus controls.

\section{Material and Methods}

The study was approved (11 June, 2015) by the ethics committee of Saarland (E242/14) and all participants gave written consent to the study $(n=193)$. They were recruited from collaborating diabetic clinics and diagnose of diabetes was made according to American Diabetes Association criteria. 186 subjects $(62 \%$ men, $38 \%$ females; mean age 64 years) were included into the study. Patient characteristics and biochemical parameters were determined from standardized clinical charts. The patients were genotyped for the Leu33Pro polymorphism using a Taqman assay modified from a previously published protocol [9]. Blood was drawn from the study participants $(n=24)$ for harvesting blood derived monocytes in Leucocept tubes (Greiner, Kremsmuenster, Austria) through Ficoll-Paque Premium density gradient centrifugation. CD14-expressing cells were selected using CD14+ micro-beads for incubation and magnetic activated cell sorting (MACS, Miltenyi biotech, Bergisch-Gladbach, Germany). The CD14+ cells were further differentiated into macrophages by incubation with X-VIVO ${ }^{\mathrm{TM}} 10$ medium containing $100 \mathrm{ng} / \mathrm{ml}$
M-CSF for 8 days. The species and vitality of the cultured cells were confirmed by immunofluorescence. Macrophages were plated in equal numbers in 12 well plates and, after serum starvation, stimulated either with $100 \mathrm{ng} / \mathrm{ml}$ LPS, $200 \mathrm{nM}$ PMA or $50 \mathrm{ng} / \mathrm{ml}$ interferon $\gamma$. MPO- and multiplex ELISAs were performed on cell supernatants according to manufacturer's instructions and normalized to cell protein concentration. Statistical methods comprised parametric and non-parametric correlation analyses, Chi-square test, ANOVA or non-parametric tests where appropriate. Statistical analyses were performed using SPSS, release 19 (IBM SPSS Statistics, Chicago).

\section{Results}

The allele frequencies were 0.84 for the wildtype and 0.16 for the Leu33Pro polymorphism. The allele frequencies were in accordance to the Hardy-Weinberg-equilibrium (Chi-square $p=0.59$ ). The characteristics of the study population according to the genotype are displayed in Table 1 . The cultivated monocytes from the diabetic patients with the different genotypes were differentiated into macrophages. Apart from morphology, we assessed the successful differentiation by verifying for expression of the macrophage specific marker EMR-1 and proper uptake of oxLDL by immunofluorescence to prove the vitality of the cultured cells (Fig. 1A, B, C). We could also show that $\beta 3$-integrin is expressed in unstimulated differentiated macrophages (Fig. 1D). We next examined the macrophage secretory capacity in response to the stimuli (LPS, PMA, Interferon $\gamma$ ) depending on the appropriate genotype. In this regard, we found no unifying and logical association of a genotype with the magnitude of any of the measured inflammatory markers.

Table 1. Clinical characteristics of patients according to the Leu33Pro polymorphism; values as mean \pm SD or median with interquartile difference.

\begin{tabular}{|c|c|c|c|}
\hline Factor & Wild-type $(n=131)$ & Heterozygous $(n=49)$ & Homozygous $(n=6)$ \\
\hline Age (years) & $65(10)$ & $62(16)$ & $70(40)$ \\
\hline BMI $\left(\mathbf{k g} / \mathbf{m}^{2}\right)$ & $29.4(6)$ & $30.0(7)$ & $30(11)$ \\
\hline Cholesterol Total (mg/dL) & $193.1 \pm 41$ & $191.6 \pm 31$ & $181.8 \pm 35$ \\
\hline HDL (mg/dl) & $46.7(21)$ & $46.2(12)$ & $46.8(32)$ \\
\hline LDL (mg/dl) & $119.8 \pm 35$ & $191.6 \pm 31$ & $181.8 \pm 35$ \\
\hline Triacylglycerol (mg/dL) & $175(114)$ & $167(149)$ & $118(59)$ \\
\hline Fasting plasma glucose $(\mathrm{mg} / \mathrm{dL})$ & $153(55)$ & $130(81)$ & $157(172)$ \\
\hline HbA1c (\%) & $7(2)$ & $7.2(2)$ & $7.5(2)$ \\
\hline CRP (mg/dL) & $0.95(3)$ & $0.28(2)$ & $0.41(0)$ \\
\hline Creatinine (mg/dL) & $0.97(0)$ & $1(0)$ & $1.32(1)$ \\
\hline GPT (U/L) & $26.5(18)$ & $28(18)$ & $18.5(9)$ \\
\hline GGT (U/L) & $35(31)$ & $27(28)$ & $32(49)$ \\
\hline Uric acid (mg/dL) & $5.9(2)$ & $5.7(2)$ & $8.3(4)$ \\
\hline RR (mmHg) & $138(26) / 80(18)$ & $139(18) / 80(4)$ & $133(43) / 82(18)$ \\
\hline GFR $\left(\mathrm{ml} / \mathrm{min} / 1,73 \mathrm{~m}^{2}\right)$ & $73.4 \pm 21$ & $81.7 \pm 22$ & $62.3 \pm 25$ \\
\hline
\end{tabular}




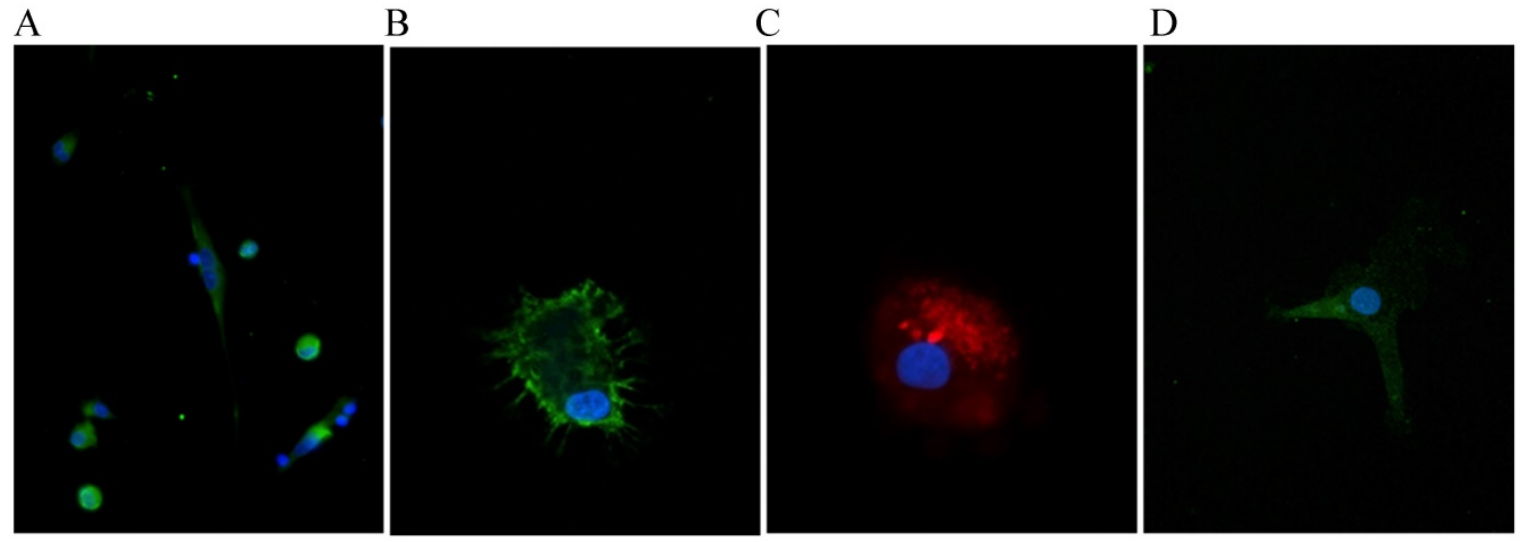

Figure 1. Representative immunofluorescent stain of macrophages for proof of appropriate differentiation: $A$ and B. Stain for nuclei (blue, DAPI) and EGF-like module-containing mucin-like hormone receptor-like 1 (green, EMR-1). C. Nucleus (blue, DAPI) and Dil-ox-LDL $20 \mu g / m l)$. D. Nucleus (blue, DAPI) and $\beta 3$-integrin (green). Imagination A: 600x, B-C: $1000 x$

\section{creatinine in the recessive model}

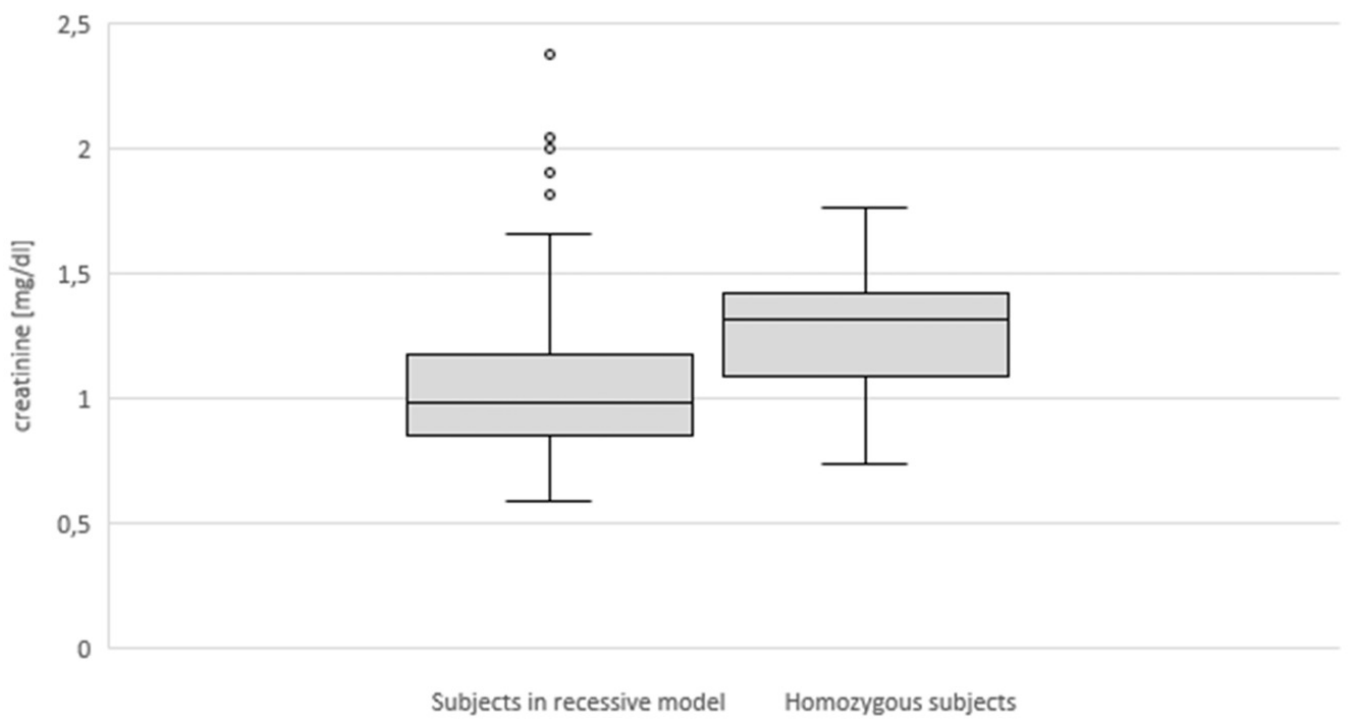

Figure 2. Genotype effect on serum creatinine in recessive model $(p<0.05)$.

Previous results suggested an association between the Leu33Pro variant in $\beta 3$-integrin with macrovascular complications in diabetic patients [10]. Here, we did not find a significant difference in the prevalence of diabetic micro- and macrovascular complications associated with the Leu33Pro polymorphism. We found that the relative frequency of diabetic nephropathy was $50 \%$ within the diabetic patients who were homozygous for the Leu33Pro polymorphism, $21 \%$ in diabetic patients homozygous for the wildtype, and $12 \%$ in the heterozygous diabetic patients. We also found indications of significant differences in GFR depending on the genotype in the overall ANOVA $(p=0.034)$ accompanied by a corresponding trend $(p=0.112)$ towards higher creatinine levels in the homozygous versus the wildtype group $[1.32 \mathrm{mg} / \mathrm{dL}(0)$ vs. 0.97 $\mathrm{mg} / \mathrm{dL}(0)]$.
Due to the overall low number of homozygous subjects in our study, we employed a recessive model to leave out one degree of freedom weight as compared to setting out 3 genotypes as separate categories. In this recessive model which requires homozygosity to show an effect we found a significant increase $(p=0.029)$ of the creatinine levels in homozygosity (Fig. 1) as well as a trend towards reduced GFR rate $(p=0.076)$ in the homozygous subjects as compared to the control group of heterozygous and wildtype patients.

\section{Conclusions}

The exact pathophysiology of the diabetes complications remains incompletely understood. In addition to the toxicity of hyperglycemia, inflammatory processes also likely make an important contribution to the pathophysiology. Especially 
macrovascular complications appear to be a result of inflammatory vascular diseases and the $\beta 3$-integrin has been implicated in inflammatory diseases and diabetes $[3,6,11]$. These observations prompted us to examine macrophages from diabetic subjects carrying the Leu33Pro allele of $\beta 3$-integrin. There are previous reports claiming that $\beta 3$-integrin is not expressed in macrophages at all [12], and yet, we were able to show that $\beta 3$-integrin is expressed in fully differentiated human macrophages (Fig. 1). To our surprise, we did not find a significantly and/or coherently altered inflammatory state in macrophages from subjects with the Leu33Pro variant of $\beta 3$-integrin. Hence, the exact function of $\beta 3$-integrin in macrophages remains to be explored as both pro-inflammatory [13] and anti-inflammatory roles are described [3] so far. We are tempted to speculate the Leu33Pro variant in $\beta 3$-integrin does not modulate the macrophages and their innate immune activity, at least not in our cohort.

The present work demonstrated homozygosity for the Leu33Pro polymorphism in $3.23 \%$ and heterozygosity in $26.34 \%$ of the diabetic subjects and thus is in accordance to previously published literature [6]. Reports on cohorts collected by other groups investigating different populations, show different allele frequencies, which suggests a significant dependence of the allele frequency on ethnicity [14].

We have found no association between the Leu33Pro variant in $\beta 3$-integrin and the diabetic state or markers of metabolism, which is also in accordance to previously published literature [7]. Our study was not designed and is too small to contribute to the large debate on the association of the Leu33Pro variant and coronary artery disease. However, we identified a trend towards the association of diabetic nephropathy and of its markers with theLeu33Pro allele in our small cohort. This finding is intriguing because $\beta 3$-integrin has thought to play a role in diabetic kidney disease for years [15]. Patients of our cohort showed a worsening of renal function with two mutated alleles of the polymorphism. Although we remain careful because of the size of our cohort and possible missed confounding factors, our results corroborate another study that showed podocyte damage in human diabetic nephropathy by activation of the $\beta 3$-integrin [16]. The mechanism by which the activation of $\beta 3$-integrin may lead to progression of diabetic kidney disease remains unclear. One could speculate that a constitutively active $\beta 3$-integrin may result in cytoskeletal re-organization leading to improper barrier function of the podocytes. Another consequence of this constitutively activate state could be a facilitation of neo-angiogenesis in the early phases of diabetic kidney disease. These speculations exceed the scope of the present work but are well corroborated by the finding that inhibition of av $\beta 3$-integrin in pigs reduced diabetic nephropathy as compared to controls [14].

In conclusion, the Leu33Pro variant of the 33 -integrin gene is associated with diabetic nephropathy suggesting that a constitutive active $\beta 3$-integrin and not the magnitude of its expression may be associated with diabetic nephropathy by complex underlying mechanistic networks. The macrophages of the Leu33Pro diabetic subjects and their inflammatory capacity do not contribute to the explanation of the observed association. The missing underlying explanation warrants further basic research efforts and epidemiological evidence.

\section{Abbreviations}

SNP: single nucleotide polymorphism; BMI: body mass index; HDL: high density lipoprotein; LDL: low density lipoprotein; SNP: single nucleotide polymorphism; GFR: glomerular infiltration rate; LPS: lipopolysaccharide; PMA : phorbol-12-myristat13-acetat; RGD: tripeptide arginine, glycine, and aspartate.

\section{Author Contributions}

$\mathrm{PH}$ and ED performed the experiments and collected the data, KR and SS contributed to the data interpretation and helped writing the paper. JGS provided the study protocol and wrote the paper. $\mathrm{PH}$ and ED contributed to the study protocol and analyzed data. All authors interpreted the findings, edited and approved the final version of the manuscript.

\section{Competing Interests}

The authors have declared that no competing interest exists.

\section{References}

1. Schneider JG, Schiekofer S, Dugi KA, et al. The proline 12 alanine substitution in the ppargamma2 gene is associated with increased extent of coronary artery disease in men. Exp Clin Endocrinol Diabetes. 2009; 117: 519-521.

2. Hynes RO, et al. Integrins: Bidirectional, allosteric signaling machines. Cell. 2002; 110: 673-687.

3. Schneider JG, Zhu Y, Semenkovich $\mathrm{CF}$, et al. Macrophage beta3 integrin suppresses hyperlipidemia-induced inflammation by modulating tnfalpha expression. Arterioscler Thromb Vasc Biol. 2007; 27: 2699-2706.

4. Su X, Esser AK, Roomp $\mathrm{K}$, et al. Antagonizing integrin $\beta 3$ increases immunosuppression in cancer. Cancer Res. 2016; 76: 3484-3495.

5. Reynolds LE, Wyder L, Hodivala-Dilke KM, et al. Enhanced pathological angiogenesis in mice lacking beta3 integrin or beta3 and beta5 integrins. Nat Med. 2002; 8: 27-34.

6. Tschoepe D, Menart B, Roesen P, et al. Genetic variation of the platelet- surface integrin gpIlib-IIIa (pia1/a2-snp) shows a high association with type 2 diabetes mellitus. Diabetologia. 2003; 46: 984-989.

7. Stratmann $\mathrm{B}, \mathrm{Xu} \mathrm{T}$, Illig $\mathrm{T}$, et al. Pla1a2 platelet polymorphism predicts mortality in prediabetic subjects of the population based kora s4-cohort. Cardiovascular diabetology. 2014; 13, 90.

8. Jallu V, Poulain P, de Brevern AG, et al. Modeling and molecular dynamics simulations of the v33 variant of the integrin subunit $\beta 3$ : Structural 
comparison with the 133 (hpa-1a) and p33 (hpa-1b) variants. Biochimie. 2014; 105: 84-90.

9. Rzezniczek S, Obuchowicz M, Pilc A, et al. Decreased sensitivity to paroxetine-induced inhibition of peripheral blood mononuclear cell growth in depressed and antidepressant treatment-resistant patients. Translational psychiatry. 2016; 6: e827.

10. Verdoia M, Secco GG, De Luca G, et al. Platelet hpa-1 a/hpa-1 b polymorphism and the risk of periprocedural myocardial infarction in patients undergoing elective pci. Platelets. 2014; 25: 367-372.

11. Maile LA, Busby WH, Patel A, et al. Blocking ligand occupancy of the av $\beta 3$ integrin inhibits the development of nephropathy in diabetic pigs. Endocrinology. 2014; 155: 4665-4675.

12. Zhao H, Ross FP, Teitelbaum SL, et al. Unoccupied alpha(v)beta3 integrin regulates osteoclast apoptosis by transmitting a positive death signal. Mol Endocrinol. 2005; 19: 771-780.

13. Antonov AS, Antonova GN, Verin AD, et al. Alphavbeta3 integrin regulates macrophage inflammatory responses via pi3-kinase/akt-dependent nf-kappab activation. J Cell Physiol. 2010; 226(2): 469-76.

14. Lim J, Lal S, Heng CK, et al. Variation of the platelet glycoprotein iiia pi(a1/a2) allele frequencies in the three ethnic groups of singapore. International journal of cardiology. 2003; 90: 269-273.

15. Jin DK, Fish AJ, Kim Y, et al. Distribution of integrin subunits in human diabetic kidneys. Journal of the American Society of Nephrology. 1996; 7: 2636-2645.

16. Staeck O, Slowinski T, Khadzhynov D, et al. Recurrent primary focal segmental glomerulosclerosis managed with intensified plasma exchange and concomitant monitoring of soluble urokinase-type plasminogen activator receptor-mediated podocyte $\beta 3$-integrin activation. Transplantation. 2015; 99: 2593-2597. 